\title{
Advances in Early Prevention of Alzheimer's Disease in the Aged
}

\author{
Liu Zhang, Yanlin Heng*, Gaijing Wang \\ Department of Medicine, Yangtze University, Jingzhou, China \\ Email: ${ }^{*} 1835323746 @ q q . c o m$
}

How to cite this paper: Zhang, L., Heng, Y.L. and Wang, G.J. (2020) Advances in Early Prevention of Alzheimer's Disease in the Aged. Yangtze Medicine, 4, 1-10. https://doi.org/10.4236/ym.2020.41001

Received: April 16, 2019

Accepted: September 14, 2019

Published: September 17, 2019

Copyright $\odot 2020$ by author(s) and Scientific Research Publishing Inc. This work is licensed under the Creative Commons Attribution International License (CC BY 4.0).

http://creativecommons.org/licenses/by/4.0/

\section{cc) (i) Open Access}

\begin{abstract}
Alzheimer's disease $(\mathrm{AD})$ is a progressive neurodegenerative disease, is one of the leading causes of death in the aging population, and has become a serious public problem endangering the physical and mental health of the elderly. The disease not only affects the health of the elderly and the quality of life in their later years, but also brings heavy financial and emotional burden to the family and society. The specific mechanism of $\mathrm{AD}$ is still unclear, and the current treatments focus on improving mental function and slowing down memory loss. The early diagnosis of $\mathrm{AD}$ is difficult and there is no cure for it, so the early prevention is particularly important. This review will introduce the major treatment, potential risk factors and summarize the relevant research about early preventions of $\mathrm{AD}$, such as regular physical sports, reasonable nutritional diet, certain social activities and so on, and expect to provide a new direction for the improvement of $\mathrm{AD}$.
\end{abstract}

\section{Keywords}

Aged, Early Prevention, Alzheimer's Disease

\section{Introduction}

\section{Overview of Alzheimer's Disease}

Alzheimer's disease is the most common type of senile dementia, characterized by the failure of memory and dementia, the onset of the disease is insidious, and the course is chronic and progressive. At present, about 47 million people suffer from dementia worldwide [1]. According to the Report of World Alzheimer, it has been estimated that the number of AD patients will increase to 65.7 million in 2030 and 150 million in 2050 if there are no effective measures. With the aggravation of aging population in China, the number of $\mathrm{AD}$ patients is the largest 
in the world and increasing every year. In 2010, the epidemiological survey showed that the incidence of $\mathrm{AD}$ in China was 5.69 million, with an annual incidence of $6.25 \%$; the elder over 55 years old are most likely to have AD [2]. Early stage of the disease can affect the daily life of patients; due to all kinds of factors, it can lead to a variety of complications or organ failure and death later [3].

AD was first proposed by Alzheimer [4] in 1907, a German neuroscientist. He pointed out that $\mathrm{AD}$ is an irreversible age-related degenerative disease of the nervous system; the main pathological markers of $\mathrm{AD}$ were nerve fiber tangles and senile plaques. In 1998, this finding was confirmed again by the Institute of Neuropathy, University of Munich, Germany. There is a large number of nerve fiber tangles and amyloid plaques, especially in the epithelium of the cerebral cortex of $\mathrm{AD}$ patients [5]. At present, the pathogenesis of $\mathrm{AD}$ is not completely clear. There are some theories are accepted by the public: the abnormal of cholinergic neurons, $\beta$-amyloid protein and its neurotoxicity, hyperphosphorylation of tau protein [6]. However, AD is a complex disease caused by genetic and environmental factors; a single hypothesis cannot fully explain its pathogenesis. Some new hypotheses, such as the hypothesis of inflammation, insulin, oxidative imbalance and gene mutation have brought into sharp focus in recent years [7].

\section{The Treatment of AD}

At present, among the five drugs approved by FDA (Food and Drug Administration) for $\mathrm{AD}$ included cholinesterase inhibitors (donepezil, rivastigmine and galantamine) and N-methyl D-aspartase NMDA receptor antagonist, but these treatments only have modest symptomatic effects for relatively short-time periods, while drugs aimed at affecting the course of disease are still in the preliminary stage of research [8]. Cholinesterase inhibitors and excitatory amino acid receptor antagonists are aimed to improve cognitive disorders of $\mathrm{AD}$; Antipsychotics, antidepressant and Benzodiazepines can effectively treat the psychobehavioral symptoms of AD patients [9]. Aducanumab (BIIB037) is a human recombinant monoclonal antibody targeting a polymerized form of $\beta$-amyloid, including soluble oligomers and insoluble fibrils deposited in amyloid plaques in the brains of $\mathrm{AD}$ patients. Biogen applied Aducanumab in clinical trials of 166 patients, showed that it could reduce amyloid plaque in patients with precursor or mild $\mathrm{AD}$ and slow down the progress of the disease, doses $30 \mathrm{mg} / \mathrm{kg}$ were generally well tolerated with no severe or serious adverse events. But its clinical effect still needs to be further studied by promoting the Aducanumab Clinical Project into Phase 3 Trials [10]. Chinese medicine including the active ingredients and compounds have been used in dementia treatment for a long history, such as huperzine A, extracts of ginkgobiloba leaves (EGb), L-3-n-butyiphathlide (L-NBP), TSH (the main water-soluble composition of our traditional Chinese medicine Polygonum multiflorum ThunbIn), ginsenosides, curcumin, Cornus officinalis 
and so on. Compared with the drugs approved by FDA, there are abundant resources of traditional Chinese medicine in China, It has unique advantages to extract effective, safe and non-toxic single traditional Chinese medicine, active ingredients and compound from natural products, but the effective ingredients of Chinese traditional medicine are complex, so it's partly difficult to extract and separate active ingredients [11]. Because the drug treatment can only delay the course of $\mathrm{AD}$, and some elderly have a high risk of taking drugs, more and more researchers focus on non-drug treatment, some small-scale randomized controlled trials have indicated that non-drug treatments have significant benefits of $\mathrm{AD}$ [12]. Noninvasive brain stimulation technology mainly includes transcranial magnetic stimulation (TMS) and transcranial direct current stimulation (tDCS). Studies indicated that TMS can effectively improve the cognitive function of $A D$ patients, especially is the high frequency magnetic stimulation; tDCS can improve AD patients' memory, but it can only stimulate a few brain tissues in the cerebral cortex and subcortex, the stimulation to deep brain tissues is weak, so the symptoms of some patients did not improve significantly [13] [14] [15]. Memory training, cognitive ability training, compensatory cognitive training, motivational interviews and error-free learning are common cognitive training methods. Compared with other non-drug therapies, these therapies are more safe and interesting, and AD patients performed high compliance, but it needs long-term persistence [16]. Study demonstrated that phototherapy can improve the sleep condition of $\mathrm{AD}$ patients by adjusting the light intensity and irradiation time, so as to improve their cognitive function [17]. Studies have shown that listening to music and singing can enhance the memory and association ability of $\mathrm{AD}$ patients, and have a significant positive effect on the improvement of mood and mental state of their caregivers [18]. For the absence of specific drugs and physiotherapy, the occurrence and development of AD can be effectively delayed by recognizing the potential risk factors of $\mathrm{AD}$ and taking early preventive measures.

\section{Potential Risk Factors of AD}

\subsection{Hereditary Risk Factors}

Apart from age, the most definite risk factor of AD is family history of dementia. Studies have shown that if there is a first-degree relative suffers from dementia, the risk of one suffers from AD will increase by $10 \%$ - 30\% [19]. Compared with the general population, someone who has at least two siblings suffers from $\mathrm{AD}$ in the family and the risk of $\mathrm{AD}$ will increase by 3 times [20]. Studies have shown that race is also an influencing factor of AD. An epidemiological study included 17,639 biological first-degree relatives of 2339 white AD patients and 2281 biological first-degree relatives of 255 African-American AD patients, the cumulative risk of dementia among African-American relatives and white relatives was $43.7 \%$ and $26.9 \%$ respectively, which showed significant difference $(\mathrm{P}<0.05)$ [21]. 


\subsection{Acquired Risk Factors}

A variety of polygenic and acquired factors may affect the occurrence of $\mathrm{AD}$, including hypertension, dyslipidemia, cerebrovascular diseases, as well as unhealthy lifestyle, environmental pollution and so on.

\subsubsection{Disease}

It's estimated that about one third of $\mathrm{AD}$ patients worldwide may be attributed to correctable risk factors, such as diabetes, middle-aged hypertension and so on [22]. Among individuals with two or more vascular risk factors in middle ages, the risk of detection of brain amyloid deposition increased to three times in later years, treating vascular risk factors in middle age is considered to be a key strategy to reduce the risk, progression and severity of $\mathrm{AD}$ [23]. 1) Hypertension: Multiple cross-sectional studies have shown that middle-aged people with hypertension are more likely to suffer from dementia. Arterial stiffness and blood pressure variability may play an important role in the association between blood pressure and AD [23] [24] [25]; 2) Dyslipidemia: study has shown that high levels of total cholesterol in middle-aged can triple the risk of $\mathrm{AD}$, it might be related to that brain cholesterol can accelerate the formation or deposition of $\beta$-amyloid protein [26]; 3) Cerebrovascular diseases: cerebrovascular diseases often coexist with $\mathrm{AD}$, and the complications of $\mathrm{AD}$ and vascular diseases are often referred to as mixed dementia. Study has shown that blood flow decreases have been observed before $\beta$-amyloid deposition in $\mathrm{AD}$ mice model, which directly contributes to amyloid deposition by impairing amyloid clearance [27].

\subsubsection{Lifestyle}

In recent years, there are enough evidence have indicated that healthy diet and lifelong learning can reduce the risk of cognitive impairment. A meta-analysis of 16 prospective studies found that the overall incidence of dementia and AD decreased by $28 \%$ and $45 \%$ in individuals with more physical activity than those with less physical activity [28]. Alzheimer's Association and the World Commission on Dementia also suggest that regular physical activity and management of cardiovascular risk factors are associated with reducing the risk of cognitive impairment [29].

\subsubsection{Environmental}

In a cross-sectional study, 2692 non-smokers aged were enrolled, the results showed that secondhand smoke exposure was associated with increasing the risk of $\mathrm{AD}$ [30]. Air pollution and pesticides are also potential risk factors of AD [31] [32].

\section{Early Prevention of AD}

\subsection{Regular Physical Exercise}

Long-term regular exercise can delay the occurrence or the progress of $\mathrm{AD}$ [33]. Wang Fang [34] found that the spatial learning and memory ability of AD mice 
model in the exercise group were significantly higher than that of the other two groups after eight weeks of aerobic treadmill exercise by studying the effect of aerobic treadmill exercise on learning and cognitive ability of $\mathrm{AD}$ mice model, aerobic exercise can effectively improve the cognitive function and delay the progress of $\mathrm{AD}$. In a cohort study, 3777 the elderly from 75 communities in southwestern France were randomly selected and followed up every two years for 15 years, the results showed that over the past 15 years, the elderly who were active in exercise and reading were less likely to suffer from dementia than those who did less exercise and reading. More exercise and reading can reduce the risk of dementia by 25\% [35]. Wang Yanping's follow-up study also indicated that less exercise could be considered as a risk factor of AD. Taking physical exercises regularly could significantly reduce the risk of mild cognitive impairment turning to $\mathrm{AD}$, which could be considered as a protective factor of $\mathrm{AD}$ [36]. A meta-analysis [37] of a series of related studies showed that short-term and medium-intensity aerobic exercise can improve cognitive function and various psycho-behavioral symptoms of $\mathrm{AD}$ patients, and then improve the quality of life. Effective way and intensity of exercise can reduce the risk of $\mathrm{AD}$, for mild cognitive impairment patients the symptoms can be improved to a certain extent and the quality of life of $\mathrm{AD}$ patients can be improved. Old people should choose the most suitable and safe exercise methods according to their age and physical strength, such as walking, jogging, Tai Chi and general daily activities such as housework, plant flowers and plants, etc.

\subsection{Rational Nutritional Diet}

Malnutrition and unreasonable diet may accelerate the development of $\mathrm{AD}$ and increase the degree of cognitive impairment. Clinical study has shown that advanced $\mathrm{AD}$ patients have different degrees of nutritional disorders [38]. Liu Xinyan et al. [39] selected $96 \mathrm{AD}$ patients and 100 the healthy and used Spearman correlation analysis and multiple regression analysis to analyze the correlation between nutritional status with $\mathrm{AD}$. The results showed that the worse the nutritional status, the worse the cognitive impairment and the self-care ability of daily life. One study explored the effect of low nutritional status on mild to moderate $\mathrm{AD}$ patients, the results showed that low levels of serum total protein and BMI were independently related to $\mathrm{AD}$, which further suggested that low nutritional level involved in the occurrence and development of AD to a certain extent [40]. One study about the correlation between AD biomarkers with BMI index showed that some $\mathrm{AD}$ biomarkers such as $\mathrm{CSFAb}$, tau and $\operatorname{tau} \mathrm{A} \beta$ were significantly correlated with BMI. Few overweight and well-nourished people were detected AD biomarkers [41]. Reasonable dietary pattern can also affect the occurrence and development of $\mathrm{AD}$. Some scholars have proposed that the deficiency of various nutrients such as $\mathrm{V}_{\mathrm{B} 1}, \mathrm{~V}_{\mathrm{B} 12}$, folic acid, and metabolic disorders are related to dementia for the impairment of brain tissue and its function [42]. Von Arnim [43] has demonstrated that malnutrition, oxidative stress and homo- 
cysteine vitamins play an important role in the pathogenesis of AD. Intaking more saturated fatty acid would increase the risk of $\mathrm{AD}$, fish oil, fish and other unsaturated fatty acids with vegetables can help the regeneration of nerve cell, prevent cognitive decline and the occurrence of AD [44]. In 2013, the Dietary Guidelines for the Prevention of Alzheimer's Disease also recommended avoiding saturated and trans fatty acids, eating more vegetables, increasing the intake of $V_{E}$ and $V_{B}$, eating more mediterranean diets such as fruits and olive oil can reduce the risk of $\mathrm{AD}$. Due to the decline of digestive, absorptive function and basic metabolism of the elderly, the utilization of nutrition in the body is insufficient, while the cognitive ability of AD patients descend. There are different degrees of mental disorders and dysphagia, $\mathrm{AD}$ patients are more prone to suffer from malnutrition than other diseases. Improving the nutritional status of patients and maintaining normal nutritional intake in the early stage of disease may be of great significance to improve the mental and behavioral symptoms of $\mathrm{AD}$ patients, so that $\mathrm{AD}$ patients can benefit from nutritional intervention.

\subsection{Social Activities}

Frequent mental training activities are of great significance in preventing $\mathrm{AD}$, such as watching TV, playing chess and playing cards can stimulate brain and reduce the risk of $\mathrm{AD}$, while little mental training can increase the probability of suffering from AD. A 26-year longitudinal study of 2513 Japanese-Americans showed that people with the lowest social participation in quartiles had a significantly increased risk of dementia, maintaining certain social ties and participating in social activities could effectively reduce the risk of dementia [45]. Scarmeas et al. [46] found that definite social activities can improve the cognitive status of AD patients, which may be related to stimulating brain cell metabolism and giving full play to brain function. The relationship between lifestyle related factors with $\mathrm{AD}$ was studied by selecting 238 patients with $\mathrm{AD}$ as case group and 476 healthy elderly as control group, family activities, keeping in touch with friends, continuing to work after retirement, tourism and active participation in social activities were included in the study criteria, then multivariate logistic regression analysis was carried out. The results showed that social activities such as playing cards, traveling, reading, exercising and maintaining good relations with family and friends were significantly correlated with $\mathrm{AD}$ [47]. Adopting a positive lifestyle and increasing the self-participation of the elderly is conducive to improving the cognitive level of AD patients. Long Yuanxian et al. [48] conducted a meta-analysis showed that the combined OR value of a certain amount of social activities was 0.48 [95\% CI $(0.25,0.92)]$, which indicated that the participation of social activities might be beneficial to train the thinking and cognitive abilities of the elderly and be a protective factor of $\mathrm{AD}$. After retirement, the elderly should keep in touch with their family and friends, participate in intellectual social activities frequently, think more, use their brains diligently and train their cognitive thinking ability, which can reduce the incidence of $\mathrm{AD}$ in the elderly. 


\section{Conclusions and Prospects}

The pathophysiological process of $\mathrm{AD}$ is thought to begin many years before the diagnosis of $\mathrm{AD}$ dementia [49]. At present, no effective detection method has been found for early diagnosis and prediction of $\mathrm{AD}$, so most $\mathrm{AD}$ patients did not receive regular examination, diagnosis and treatment [50]. The emotional, mental and behavioral symptoms of AD patients during the whole course of the disease have brought tremendous psychological and economic burden to the patients and their families. Identifying potential risk factors and taking early prevention measures, including appropriate sports, reasonable nutritional diet and certain social activities can slow down the occurrence and development of AD. However, systematic and comprehensive prevention guidelines have not yet been formulated. In addition to continuing to study these mechanisms, prevention and treatment of $\mathrm{AD}$ by these interventions are also needed. Whether there is gender difference in the improvement effect, whether it can prevent the occurrence of $\mathrm{AD}$ and its combined treatment with drugs should be further discussed.

\section{Conflicts of Interest}

The authors declare no conflicts of interest regarding the publication of this paper.

\section{References}

[1] World Alzheimer Report 2015: The Global Impact of Dementia.

[2] Zhang, G., Ding, H., Chen, H., et al. (2013) Thiamine Nutritional Status and Depressive Symptoms Are Inversely Associated among Older Chinese Adults. Journal of Nutrition, 143, 53-58. https://doi.org/10.3945/jn.112.167007

[3] Zhao, G. (2012) Exercise, Alzheimer's Disease and Synaptic Plasticity (Review). Chinese Journal of Rehabilitation Theory and Practice, 18, 244-246.

[4] Alzheimer, A. (1907) Uber Eine Eigenartige Erkrankung Der Hirnrinde. Allgemei Ne Zeitschriftfur Psychiastre and Psychsch Gerichtlich Medizine, 64, 146-148.

[5] Graaeber, M.B., Kosel, S., Grasbon Frodl, E., et al. (1998) Histopathology and APOE Genotype of the First Alzheimer Disease Patient, Auguste D. Neurogenetics, 1, 223-228. https://doi.org/10.1007/s100480050033

[6] Khairallah, M.I. and Kassem, L.A.A. (2011) Alzheimer's Disease: Current Status of Etiopathogenesis and Therapeutic Strategies. Pakistan Journal of Biological Science, 14, 257-272. https://doi.org/10.3923/pjbs.2011.257.272

[7] Ying, X., Wu, Z., Lei, Y., et al. (2014) Research Progress on Pathogenesis and Therapeutic Drugs of Alzheimer's Disease. China Pharmacy, 25, 3152-3155.

[8] Zhu, J., Zhang, Z., Dong, Y.X., et al. (2018) Recent Advances in Alzheimer's Disease and Its Drug Discovery. Chinese Pharmacological Bulletin, 34, 1629-1634.

[9] Jia, J.P., Xing, Y., Wu, L.Y., et al. (2014) Guidelines for the Diagnosis and Treatment of Alzheimer's Disease. Zhejiang Medical Journal, 36, 1127-1128.

[10] Ferrrero, J., Williams, L., Stella, H., et al. (2016) First-in-Human, Double-Blind, Placebo-Controlled, Single-Dose Escalation Study of Aducanumab (BIIB037) in Mild-to-Moderate Alzheimer's Disease. Alzheimer's \& Dementia, 2, 169-176. https://doi.org/10.1016/j.trci.2016.06.002 
[11] Li, L., Wang, X.L. and Peng, Y. (2016) Pharmacological Research of Natural Products in the Treatment of Alzheimer's Disease. Chinese Pharmacological Bulletin, 32, 149-155.

[12] Lu, S.Y. and Xu, B. (2019) Progress in the Treatment of Alzheimer's Disease. Medical Journal of the Chinese People's Armed Police Force, 30, 268-272.

[13] Hsu, W.Y., Ku, Y., Zanto, T.P., et al. (2015) Effects of Noninvasive Brain Stimulation on Cognitive Function in Healthy Aging and Alzheimer's Disease: A Systematic Review and Meta-Analysis. Neurobiology of Aging, 36, 2348-2359. https://doi.org/10.1016/j.neurobiolaging.2015.04.016

[14] Zhang, C., Hu, W.H., Wu, D.L., et al. (2015) Behavioral Effects of Deep Brain Stimulation of the Anterior Nucleus of Thalamus, Entorhinal Cortex and Fornix in a Rat Model of Alzheimer's Disease. Chinese Medical Journal, 128, 1190-1195. https://doi.org/10.4103/0366-6999.156114

[15] Tremblay, S., Lepage, J.F., Latulipe-Loiselle, A., et al. (2014) The Uncertain Outcome of Prefrontal tDCS. Brain Stimulation, 7, 773-783. https://doi.org/10.1016/j.brs.2014.10.003

[16] Choi, J. and Twamley, E.W. (2013) Cognitive Rehabilitation Therapies for Alzheimer's Disease: A Review of Methods to Improve Treatment Engagement and Self-Efficacy. Neuropsychology Review, 23, 48-62. https://doi.org/10.1007/s11065-013-9227-4

[17] Figueiro, M.G., Plitnick, B.A., Lok, A., et al. (2014) Tailored Lighting Intervention Improves Measures of Sleep, Depression, and Agitation in Persons with Alzheimer's Disease and Related Dementia Living in Long-Term Care Facilities. Clinical Interventions in Aging, 9, 1527-1537. https://doi.org/10.2147/CIA.S68557

[18] Osman, S.E., Tischler, V. and Schneider, J. (2016) "Singing for the Brain": A Qualitative Study Exploring the Health and Well-Being Benefits of Singing for People with Dementia and Their Careers. Dementia (London), 15, 1326-1339. https://doi.org/10.1177/1471301214556291

[19] Van Duiji, C.M., Clayton, D., Chandra, V., et al. (1991) Familial Aggregation of Alzheimer's Disease and Related Disorders: A Collaborative Re-Analysis of Case-Control Studies. International Journal of Epidemiology, 20, S13-S20. https://doi.org/10.1093/ije/20.Supplement_2.S13

[20] Vardarajan, B.N., Faber, K.M., Bird, T.D., et al. (2014) Age-Specific Incidence Rates for Dementia and Alzheimer Disease in NIA-LOAD/NCRAD and EFIGA Families: National Institute on Aging Genetics Initiative for Late-Onset Alzheimer Disease/National Cell Repository for Alzheimer Disease (NIA-LOAD/NCRAD) and Estudio Familiar de Influencia Genetica en Alzheimer (EFIGA). The Journal of the Medical Association, 71, 315-323. https://doi.org/10.1001/jamaneurol.2013.5570

[21] Green, R.C., Cupples, L.A., Go, R., et al. (2002) Risk of Dementia among White and African American Relatives of Patients with Alzheimer Disease. The Journal of the Medical Association, 287, 329-336. https://doi.org/10.1001/jama.287.3.329

[22] Norton, S., Matthews, F.E., Barnes, D.E., et al. (2014) Potential for Primary Prevention of Alzheimer's Disease: An Analysis of Population-Based Data. The Lancet Neurology, 13, 788-794. https://doi.org/10.1016/S1474-4422(14)70136-X

[23] Gottesman, R.F., Albert, M.S., Alonso, A., et al. (2017) Association between Midlife Vascular Risk Factors and Estimated Brain Amyloid Deposition. The Journal of the Medical Association, 317, 1443-1450. https://doi.org/10.1001/jama.2017.3090

[24] Gilsan, P., Mayeda, E.R., Glymour, M.M., et al. (2017) Female Sex, Early-Onset Hypertension, and Risk of Dementia. Neurology, 89, 1886-1893. 
https://doi.org/10.1212/WNL.0000000000004602

[25] Oishi, E., Ohara, T., Sakata, S., et al. (2017) Day-to-Day Blood Pressure Variability and Risk of Dementia in a General Japanese Elderly Population: The Hisayama Study. Circulation, 136, 516-525. https://doi.org/10.1161/CIRCULATIONAHA.116.025667

[26] Kivipelto, M., Helkala, E.L., Laalso, M.P., et al. (2002) Apolipoprotein E Epsilon4 Allele, Elevated Midlife Total Cholesterol Level, and High Midlife Systolic Blood Pressure Are Independent Risk Factors for Late-Life Alzheimer Disease. Annals of Internal Medicine, 137, 149-155. https://doi.org/10.7326/0003-4819-137-3-200208060-00006

[27] Ighodaro, E.T., Abner, E.L., Fardo, D.W., et al. (2017) Risk Factors and Global Cognitive Status Related to Brain Arteriolosclerosis in Elderly Individuals. Journal of Cerebral Blood Flow \& Metabolism, 37, 201-216. https://doi.org/10.1177/0271678X15621574

[28] Hamer, M. and Chida, Y. (2009) Physical Activity and Risk of Neurodegenerative Disease: A Systematic Review of Prospective Evidence. Psychological Medicine, 39, 3-11. https://doi.org/10.1017/S0033291708003681

[29] Baumgart, M., Snyder, H.M., Carrillo, M.C., et al. (2015) Summary of the Evidence on Modifiable Risk Factors for Cognitive Decline and Dementia: A Population-Based Perspective. Alzheimer's Dementia, 11, 718-726. https://doi.org/10.1016/j.jalz.2015.05.016

[30] Chen, R. (2012) Association of Environmental Tobacco Smoke with Dementia and Alzheimer's Disease among Never Smokers. Alzheimer's Dementia, 8, 590-595. https://doi.org/10.1016/j.jalz.2011.09.231

[31] Reed, W., Maronpot, R.R., et al. (2004) Brain Inflammation and Alzheimer's-Like Pathology in Individuals Exposed to Severe Air Pollution. Toxicological Pathology, 32, 650-658. https://doi.org/10.1080/01926230490520232

[32] Richardson, J.R., Roy, A., Shalat, S.L., et al. (2014) Elevated Serum Pesticide Levels and Risk for Alzheimer Disease. JAMA Neurology, 71, 284-290. https://doi.org/10.1001/jamaneurol.2013.6030

[33] Chan, K.Y., Wang, W., Wu, J.J., et al. (2013) Epidemioligy of Alzheimer's Disease and Other Forms of Dementia in China, 1990-2010: A Systematic Review and Analysis. The Lancet, 381, 2016-2023. https://doi.org/10.1016/S0140-6736(13)60221-4

[34] Wang, F. (2011) Effects of Aerobic Exercise on Learning and Memory, a $\beta$-Related Gene Expression and Lipid Peroxidative of AD Rats. East China Normal University, Shanghai.

[35] Le Goff, M., Helmer, C., Foubert-Samier, A., et al. (2009) Activities in Retired People and the Risk of Dementia. Comptes Rendus Biologies, 332, 378-384. https://doi.org/10.1016/j.crvi.2008.11.006

[36] Wang, Y.P. (2011) The Study on Prevalence and Incidence of Alzheimer's Disease and the Analysis of Influencing Factors in Taiyuan. Shanxi Medical University, Taiyuan.

[37] Yang, S.S., Yang, X.J., Li, M., et al. (2017) The Intervention Effectiveness of Moderate Aerobic Exercise for Patients with Alzheimer's Disease: A Meta-Analysis. Chinese Journal of Nursing, 52, 686-691.

[38] Cunnane, S.C., Schneider, J.A., Tangney, C., et al. (2010) Plasma and Brain Fatty Acid Profiles in Mild Cognitive Impairment and Alzheimer's Disease. Journal of 
Alzheimer's Disease, 293, 691-697. https://doi.org/10.3233/JAD-2012-110629

[39] Liu, X.Y., Liu, W.Y., Yu, X.J., et al. (2014) Study on Correlation between Nutritional Status and Alzheimer's Disease. China Medical Herald, 11, 62-65.

[40] Wang, G.H., Chen, Q., Zhao, X.B., et al. (2017) Effect of Lower Nutritional State on Mild to Moderate Alzheimer's Disease with Positive $\beta$-Amyloid Plaque Imaging. Chinese Journal of Geriatric Heart Brain and Vessel Disease, 19, 345-348.

[41] Vidoni, E.D., Townley, R.A., et al. (2011) Alzheimer's Disease Biomarkers Are Associated with Body Mass Index. Neurology, 77, 1920-1931. https://doi.org/10.1212/WNL.0b013e318238eec1

[42] Guigoz, Y. (2006) The Mini-Nutritional Assessment (MNA) Review of the Literature What Does It Tell Us? Journal of Nutrition Health Aging, 10, 466-485.

[43] Von Arnim, C.A., Gola, U., et al. (2010) More than the Sum of Its Parts? Nutrition in Alzheimer's Disease. Nutrition, 26, 694-700. https://doi.org/10.1016/j.nut.2009.11.009

[44] Hu, F.B. and Willet, W.C. (2002) Optimal Diets for Prevention of Coronary Heart Disease. JAMA, 288, 2569-2578. https://doi.org/10.1001/jama.288.20.2569

[45] Saczynski, J.S., Pfeifer, L.A., et al. (2006) The Effect of Social Engagement on Incident Dementia. American Journal of Epidemiology, 163, 433-440. https://doi.org/10.1093/aje/kwj061

[46] Scarmeas, N., Luchsinger, J.A., Schupt, N., et al. (2009) Physical Activity, Diet, and Risk of Alzheimer's Disease. JAMA, 302, 627-637. https://doi.org/10.1001/jama.2009.1144

[47] Zhou, D.S., Xu, Y.E., Yu, C., et al. (2014) A Case-Control Study on the Relationship between Lifestyle and Alzheimer's Disease in the Elderly. 7 th National Conference on Mental Health, China, 76-80.

[48] Long, Y.X., Tang, Y.M. and Tang, L.J. (2013) Meta-Analysis on Major Risk Factors of Alzheimer's Disease in China. Chinese Preventive Medicine, 14, 59-63.

[49] Reisa, A., Sperling, P.S., et al. (2011) Toward Defining the Preclinical Stages of Alzheimer's Disease: Recommendations from the National Institute on Aging-Alzheimer's Association Workgroups on Diagnostic Guidelines for Alzheimer's Disease. Alzheimer's and Dementia, 7, 280-292. https://doi.org/10.1016/j.jalz.2011.03.003

[50] Sun, M.S. and Gu, M.M. (2018) Research Progress in Early Diagnosis of Alzheimer's Disease. Chinese Journal of Contemporary Neurology and Neurosurgery, 18, 213-221. 